\title{
Long noncoding RNA NEATI promotes cell proliferation and invasion by regulating hnRNPA2 expression in hepatocellular carcinoma cells
}

This article was published in the following Dove Press journal:

OncoTargets and Therapy

20 February 2017

Number of times this article has been viewed

\author{
Yuanyi Mang \\ Li Li \\ Jianghua Ran \\ Shengning Zhang \\ Jing Liu \\ Laibang Li \\ Yiming Chen \\ Jian Liu \\ Yang Gao \\ Gang Ren
}

Department of Hepato-BiliaryPancreatic Surgery, The Calmette Affiliated Hospital of Kunming Medical University, The First Hospital of Kunming, Kunming, Yunnan, People's Republic of China
Correspondence: Li Li

Department of Hepato-Biliary-Pancreatic Surgery, The Calmette Affiliated Hospital of Kunming Medical University, The First Hospital of Kunming, 1228 Beijing Road, Kunming, 650000 Yunnan, People's Republic of China Email ynkmlili62@hotmail.com

\begin{abstract}
Growing evidence demonstrates that long noncoding RNAs (lncRNAs) are involved in the progression of various cancers, including hepatocellular carcinoma (HCC). The role of nuclear-enriched abundant transcript 1 (NEAT1), an essential lncRNA for the formation of nuclear body paraspeckles, has not been fully explored in HCC. We aimed to determine the expression, roles and functional mechanisms of NEAT1 in the proliferation and invasion of HCC. Based on real-time polymerase chain reaction data, we suggest that $N E A T 1$ is upregulated in HCC tissues compared with noncancerous liver tissues. The knockdown of NEAT1 altered global gene expression patterns and reduced HCC cell proliferation, invasion and migration. RNA immunoprecipitation and RNA pull-down assays confirmed that U2AF65 binds to NEAT1. Furthermore, the study indicated that NEAT1 regulated $h n R N P A 2$ expression and that this regulation may be associated with the NEAT1-U2AF65 protein complex. Thus, the NEAT1hnRNP $A 2$ regulation mechanism promotes HCC pathogenesis and may provide a potential target for the prognosis and treatment of HCC.
\end{abstract}

Keywords: long noncoding RNA, NEATl, RNA-binding protein, HCC

\section{Introduction}

Long noncoding RNAs (lncRNAs) are a new class of noncoding RNAs that are longer than 200 nucleotides. These RNAs have been found to be frequently dysregulated in various diseases and play an essential role in imprinting control, cell differentiation, immune responses, human diseases, tumorigenesis and other biologic processes. ${ }^{1-3}$ Understanding the lncRNAs that function in various diseases and their precise molecular mechanisms will be critical for exploring potential strategies for early diagnosis and therapy. ${ }^{4}$ Recent studies have indicated that lncRNA-protein complexes can give rise to unique transcriptional programs, which result in different functions based on the interactions between the bound protein and RNA. ${ }^{5-7}$ Genetic studies have proven that a significant number of lncRNAs are associated with hepatocellular carcinoma (HCC) and that the interaction between IncRNAs and RNA-binding proteins is involved in HCC tumorigenicity. ${ }^{8,9}$

The lncRNA nuclear-enriched abundant transcript 1 (IncRNA-NEAT1) is transcribed from the familial tumor syndrome multiple endocrine neoplasia type 1 locus on chromosome 11 and lacks any introns. ${ }^{10,11}$ NEAT1 is retained in the nucleus, where it forms the core structural component of paraspeckle suborganelles and acts as a transcriptional regulator for numerous genes, including the genes involved in cancer progression. ${ }^{12}$ Aberrant NEAT1 expression has been reported in several human malignancies, including 
leukemia, ${ }^{13}$ glioma, ${ }^{14}$ non-small cell lung cancer, ${ }^{15}$ prostate cancer, ${ }^{16}$ breast cancer ${ }^{17}$ and ovarian carcinoma. ${ }^{18}$ NEAT1 plays important roles in tumorigenesis; specifically, it is involved in the cooperation of multiple paraspeckle-localized RNA-binding proteins, including splicing factor family proteins, ${ }^{19}$ and it regulates messenger RNA (mRNA) export ${ }^{12}$ and controls target gene transcription by protein sequestration into paraspeckles. ${ }^{20}$ A recent study analyzing multiple samples indicated that the expression level of NEAT1 strongly correlates with the number of tumor nodes, metastasis and TNM stage in HCC patients. ${ }^{21}$ However, the role of NEAT1 as a driver of this regulation and its function in these molecular mechanisms remains unclear in HCC.

In this study, we found that NEAT1 was upregulated in $\mathrm{HCC}$ tissues and cell lines. Furthermore, the upregulation of NEAT1 promoted cell proliferation, migration and invasion in HCC cell lines. To focus on the function and potential molecular mechanism of NEAT1 in HCC, we profiled its gene expression pattern by gene sequencing in a NEAT1knockdown HepG2 cell line and verified by reverse transcription quantitative polymerase chain reaction (RT-qPCR) that heterogeneous nuclear ribonucleoprotein A2 (hnRNP A2) and IQGAPI were downregulated and signal transducer and activator of transcription 1 (STAT1), oncostatin $\mathrm{M}$ receptor $(O S M R)$ and insulin-like growth factor binding protein 3 (IGFBP3) were upregulated. Based on RNA-protein interaction data obtained from starbase 2.0, RNA immunoprecipitation (RIP) and RNA pull-down assays, we verified that U2 small nuclear RNA auxiliary factor 2 (U2AF65) protein binds to NEAT1. Furthermore, we confirmed that NEAT1 functioned by regulating $h n R N P A 2$ expression, which might be associated with the NEAT1-U2AF65 protein complex.

\section{Materials and methods Patients and cell lines}

Twelve clinical tumor samples were collected from surgical resections of liver tumors conducted at the Department of Hepatobiliary Surgery of the The Calmette Affiliated Hospital of Kunming Medical University, The First Hospital of Kunming, Kunming, Yunnan, People's Republic of China). Adjacent normal tissues, which are defined as normal in the results, were obtained $2 \mathrm{~cm}$ distal from the HCC tissue. These tissues were divided into two groups according to the 2003 American Joint Committee on Cancer TNM classification: grades I-II HCC group ( $\mathrm{n}=5)$ and grades III-IV HCC group $(n=7)$. All patients provided written informed consent, and ethical consent for this study was granted by the Committee for Ethical Review of Research Involving Human Subjects of the The Calmette Affiliated Hospital of Kunming Medical
University, The First Hospital of Kunming. The HCC cell lines HepG2, SMMC-7721 and HCCLM3 were purchased from American Type Culture Collection (Manassas, VA, USA). All HCC cells were maintained in Dulbecco's Modified Eagle's Medium (DMEM) supplemented with $10 \%$ fetal bovine serum, $100 \mathrm{U} / \mathrm{mL}$ penicillin and $100 \mu \mathrm{g} / \mathrm{mL}$ streptomycin.

\section{Cell transfection}

All constructs used to knock down NEAT1 were purchased from RiboBio (People's Republic of China). After $24 \mathrm{~h}$ of culture, the HepG2 and SMMC-7721 cells were transfected using Opti-MEM I and Lipofectamine 2000 (Thermo Fisher Scientific, Waltham, MA, USA) for $6 \mathrm{~h}$ at approximately $70 \%$ confluence according to the manufacturer's instructions. The effect of knockdown was detected by RT-qPCR $48 \mathrm{~h}$ after transfection. NEAT1 was stably knocked down by selection with gentamicin (G418; Thermo Fisher Scientific) as described previously. ${ }^{14}$ Cells were stably transduced with retroviral vectors expressing T7-tagged $h n R N P A 2$ complementary DNA (cDNA) to overexpress $h n R N P A 2$ as described previously. ${ }^{22}$ The following small interfering RNAs (si-RNAs) were used to knock down expression:

Si-NEAT1: Target: GCCTCCGGTCATACTAGTT

Forward: 5'-GCCUCCGGUCAUACUAGUU dTdT-3'

Reverse: 3'-dTdT CGGAGGCCAGUAUGAUCAA-5'

Si-U2AF65: Target: CCAACTACCTGAACGATGA

Forward: 5'-CCAACUACCUGAACGAUGA dTdT-3'

Reverse: $3^{\prime}$-dTdT GGUUGAUGGACUUGCUACU-5'

Si-NC: Forward: 5'-UUCUCCGAACGUGUCACG UTT-3'

Reverse: 5'-ACGUGACACGUUCGGAGAATT-3'.

\section{RNA extraction and RT-qPCR}

Total RNA was extracted from the cell lines using TRIzol reagent. RNA was then transcribed into cDNA using a High-Capacity cDNA Reverse Transcription Kit (Thermo Fisher Scientific) and a reaction volume of $20 \mu \mathrm{L}$. Realtime polymerase chain reactions (real-time PCRs) were conducted in a $20 \mu \mathrm{L}$ reaction volume on a Thermo Fisher Scientific 7500 using an SYBR Green mix. The expression of each RNA was normalized to that of glyceraldehyde 3-phosphate dehydrogenase (GAPDH), and fold changes in expression were calculated using the $2^{-\Delta \Delta \mathrm{Ct}}$ method. The following primers were used in this study: NEAT1: Forward: GUCUGUGUGGAAGGAGGAATT

Reverse: UUCCUCCUUCCACACAGACTT

hnRNP A2: Forward: CAGCAACCTTCTAACTA CGGTCC 
Reverse: CTGCCTCCTGGACCATAGTTTC

GAPDH: Forward: GAACGGGAAGCTCACTGG

Reverse: GCCTGCTTCACCACCTTCT-3'

IQGAP 1: Forward: GGTTATCACCCTCATTCGTTC

Reverse: TTTCCTCTTGGAGTGCTGTCT

STAT1: Forward: CAGAAATGTGAAGGA CAAGGTT

Reverse: GATAGGGTCATGTTCGTAGGTG

OSMR: Forward: TGCTTCTCCTGCTTCTGTAATA

Reverse: TGCTTCTCCTGCTTCTGTAATA

IGFBP3: Forward: AGCTCCAGGAAATGCTAGTGA

Reverse: AGGCTGCCCATACTTATCCAC.

\section{Cell proliferation assay}

(carboxyfluorescein diacetate, succinimidyl ester-labeled assay and cell counting kit-8 assay)

A total of $2 \times 10^{5}$ HepG2 or SMMC-7721 cells labeled with $2 \mu \mathrm{M}$ carboxyfluorescein diacetate, succinimidyl ester (CFSE) were seeded in six-well plates $48 \mathrm{~h}$ after si-NEAT1 or si-NC treatment. The cells were collected, and the CFSE intensity was detected by flow cytometry to measure cell proliferation at 0 and $72 \mathrm{~h}$ after seeding. Forty-eight hours after the si-NEAT1 or si-NC treatment, the cells were seeded in 96-well plates at a density of 2,000 cells per well. A cell counting kit-8 (CCK-8) assay was used to assess cell viability at $0,12,24$ and $48 \mathrm{~h}$ after seeding. The absorbance was measured at $450 \mathrm{~nm}$ using an automatic microplate reader.

\section{Analysis of invasiveness and mobility}

The invasive and migratory potentials were assessed with an in vitro Transwell (EMD Millipore, Billerica, MA, USA) assay as previously described. ${ }^{23}$ Briefly, a total of $2 \times 10^{5}$ HepG2 or SMMC-7721 cells suspended in $200 \mu \mathrm{L}$ of serumfree DMEM were seeded in the upper chamber, whose porous membrane was coated with Matrigel (BD Biosciences; $200 \mu \mathrm{g} / \mathrm{mL}, 100 \mu \mathrm{L}$ ) for the Transwell invasion assay; the membrane remained uncoated for the migration assay. Subsequently, serum was added to the lower chamber (final concentration: $15 \%$ ) as a chemoattractant. After migration for $24 \mathrm{~h}$ or invasion for $48 \mathrm{~h}$, cells that had penetrated the filters were fixed in dry methanol, stained with $0.1 \%$ crystal violet and then photographed. The cells on the filters in each group were detached by trypsin. The cells were then counted using a hemocytometer.

\section{RNA sequencing and data analysis}

HepG2 cells were transfected with si-NEAT1 or si-NC in duplicate. Forty-eight hours after transfection, total RNA was isolated from the cells using TRIzol (Thermo Fisher Scientific) according to the manufacturer's protocol. RNA purity was assessed using an ND-1000 Nanodrop instrument. The A260:A280 and A260:A230 ratios of each RNA sample exceeded 1.8 and 2.0, respectively. RNA integrity was evaluated using an Agilent 2200 TapeStation (Agilent Technologies, Santa Clara, CA, USA), and the RNA integrity number evaluation of each sample exceeded 7.0. Briefly, mRNAs were isolated from the total RNA and fragmented to a size of approximately $200 \mathrm{bp}$. Subsequently, second strand of cDNA was synthesized, followed by adaptor ligation and enrichment with a low cycle according to the instructions of the TruSeq ${ }^{\circledR}$ RNA LT/HT Sample Prep Kit (Illumina, San Diego, CA, USA). The purified library products were evaluated using the Agilent 2200 TapeStation and Qubit ${ }^{\circledR} 2.0$ (Thermo Fisher Scientific). The samples were then diluted to $10 \mathrm{pM}$ for cluster generation in situ on the HiSeq 2500 pair-end flow cell, followed by sequencing ( $2 \times 100 \mathrm{bp}$ ) on HiSeq 2500 . The transformed data of the two groups were compared using Welch's $t$-test. The threshold for up- and downregulated genes was defined as a fold change $>2.0$ and a $P$-value $<0.05$. Geneontology and Kyoto Encyclopedia of Genes and Genomes analyses were employed to determine the roles of these differentially expressed mRNAs.

\section{RNA transcription in vitro}

The target fragments of RNAs were transcribed using the MEGAscript Kit (Ambion). The DNA templates used for transcription were cloned using a TOPO TA Cloning Kit (Invitrogen). The following primers were used for the RT-qPCR:

Fragment 1 of NEAT1: Forward: CTGAGTTAGAT GAGACGAGGGG; Reverse: CTGGCATGGACA AGTTGAAGA

Fragment 2 of NEAT1: Forward: CCTAGCATGT TTGACAGGCG; Reverse: AATGCTAGGACTC ACACTGGC

Fragment 3 of NEAT1: Forward: CTGTATTCAGGA GGCTACCATT; Reverse: AACGCCCCAAGTTAT TTCATC

Fragment 4 of NEATl: Forward: AAGGTGGGGAAG ACTGAAGAA; Reverse: AGGAACAAATCCAGA AGAGCC

Fragment 5 of NEAT1: Forward: AGCCAAGACTAG AGGGGAAAC; Reverse: ACAACAGCATACCC GAGACTAC.

\section{RNA pulldown}

An RNA pull-down analysis was performed as previously described. ${ }^{7}$ Briefly, biotinylated NEATI or RNA fragments 
were incubated with cell protein extracts $(8 \mu \mathrm{g})$, which were then targeted with streptavidin beads and washed. The bound proteins were resolved by gel electrophoresis. The specific bands were excised and identified by Western blotting. Additional details are described in the figure legends.

\section{RNA immunoprecipitation}

RIP experiments were performed using the Magna RIP RNABinding Protein Immunoprecipitation Kit (EMD Millipore) according to the manufacturer's instructions. Additional details are described in the figure legends.

\section{Electrophoretic mobility shift assay (EMSA)}

EMSA experiments were performed using a Light Shift Chemiluminescent RNA EMSA Kit. The target RNAs were labeled with the Pierce ${ }^{\mathrm{TM}}$ RNA 3' End Biotinylation Kit (Thermo Fisher Scientific). A total of $20 \mu \mathrm{L}$ containing cell extracts, labeled RNA and unlabeled RNA were mixed as described in the figure legends. The masses of these systems were resolved using $6 \%$ native polyacrylamide gel electrophoresis. Then, they were transmembraned and photographed by charge coupled device camera using chemiDoc XRS (Bio Rad).

\section{In vivo tumor xenograft studies}

The stably transfected cell lines were generated as described previously. ${ }^{24}$ The axillary fossae of male athymic nude mice aged 4-6 weeks were then bilaterally inoculated with $2 \times 10^{6}$ stably overexpressing si-NEAT1 HepG2 cells or control cells per site. Tumor size was monitored by measuring the length and width with calipers, and the volumes were calculated with the following formula: $(\mathrm{L} \times \mathrm{W} 2) \times 0.5$, where $\mathrm{L}$ is the length and $\mathrm{W}$ is the width of each tumor. The mice used in this experiment were purchased from Kunming Medical University and maintained under specific pathogen-free conditions at Kunming Medical University in Kunming, Yunnan, People's Republic of China. All mouse studies were performed in compliance with the Institutional Animal Care and Use Committee policies and the guidelines of Kunming Medical University.

\section{Statistical analysis}

All data are presented as the mean \pm standard deviation (SD). The data are displayed in graphs, and significance of differences was assessed using GraphPad 5.0 software. Flow cytometry data were analyzed with Flowjo software.

\section{Results \\ NEATI is upregulated in HCC tissues and cell lines}

The expression level of NEAT1 was analyzed in $12 \mathrm{HCC}$ tissues, three adjacent normal tissues, and the HepG2, SMMC-7721 and HCCLM3 cell lines using RT-qPCR. $\mathrm{HCC}$ tissues and cell lines expressed higher levels of NEAT1, compared with adjacent normal tissues (Figure 1A).
A

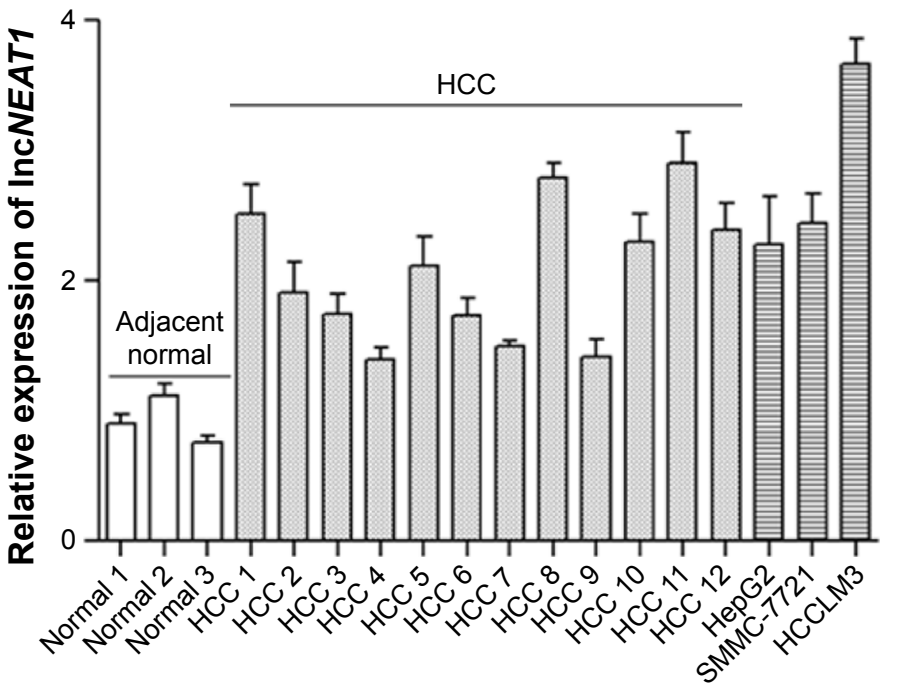

B

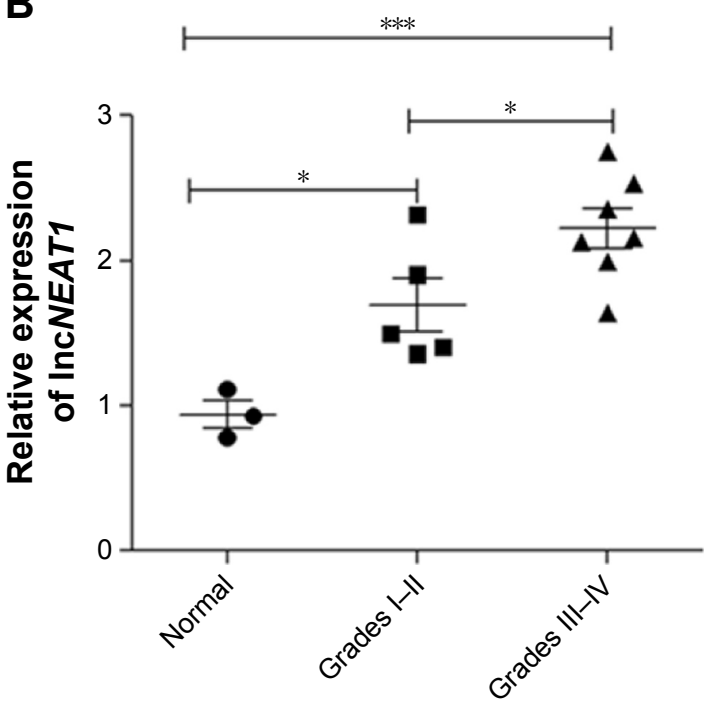

Figure I The expression of NEATI is upregulated in HCC tissues and cell lines.

Notes: (A) Quantitative analysis of the expression levels of NEATI in samples normalized to GAPDH according to the $2^{-\Delta \Delta C \mathrm{t}}$ method. Three adjacent normal tissues, I2 HCC tissues and the HCC cell lines HepG2, SMMC-772I and HCCLM3 were analyzed. (B) The statistical graph shows the quantitative analysis of the expression levels of NEATI in adjacent normal tissues, low-grade HCC (I-II) and high-grade HCC (III-IV). The significance of differences in each group was measured with a one-way ANOVA followed by Dunnett's post hoc multiple comparison test. $* P<0.05$, ***P<0.001.

Abbreviations: ANOVA, analysis of variance; HCC, hepatocellular carcinoma; GAPDH, lyceraldehyde 3-phosphate dehydrogenase; NEATI, nuclear-enriched abundant transcript I. 
Furthermore, the $12 \mathrm{HCC}$ tissues, as shown in Figure 1A, were classified as low-grade HCC (I-II) and high-grade HCC (III-IV) according to the TNM stage, which was determined by the pathologic diagnosis and the clinical status. The data indicated that NEAT1 expression directly correlated with the pathologic grade of HCC (Figure 1B).

\section{Knockdown of NEATI inhibited the proliferation, migration and invasion of HCC cells}

To assess the function of NEAT1 in HCC cells, the cells were transfected with small interfering RNA (siRNA) against
NEAT1, si-NEAT1, and si-NC was used as a control. The efficacy of siRNA knockdown was measured in HCC cell lines (approximately 70\% decrease in HepG 2 cells and 60\% decrease in SMMC-7721 cells; Figure 2A). Proliferation was assessed with CFSE labeling and a CCK-8 assay, and these experiments indicated that the knockdown of NEAT1 significantly inhibited the proliferation of HepG2 and SMMC-7721 cells, compared with the si-NC group $(P<0.01$; Figure 2B, C). Transwell assays were used to detect the effect of si-NEAT1 on the invasiveness and migration of HCC cells. As shown in Figure 3A, the knockdown of NEAT1 reduced the invasiveness and migration of HepG2 and SMMC-7721

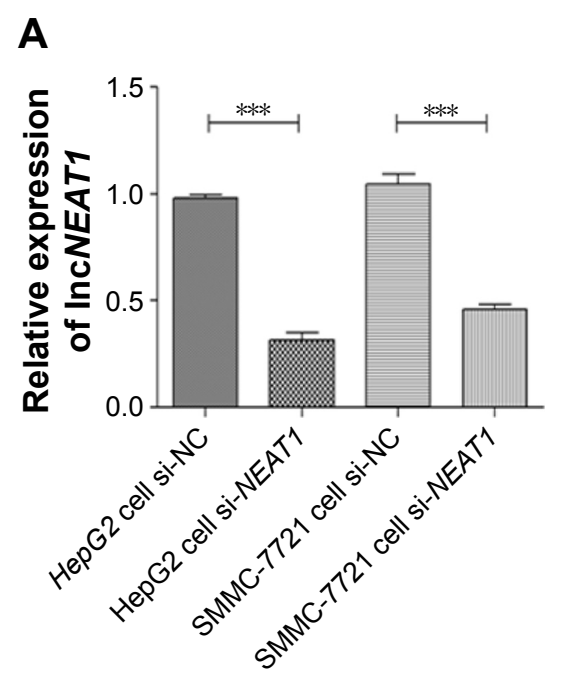

B

HepG2

SMMC-7721
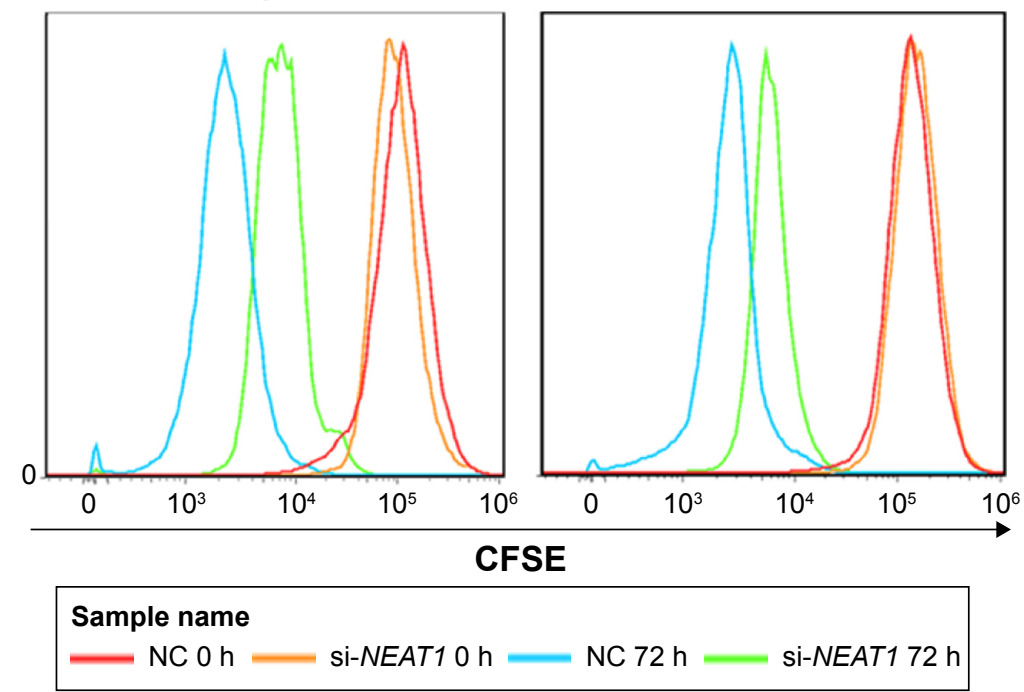

C

HepG2

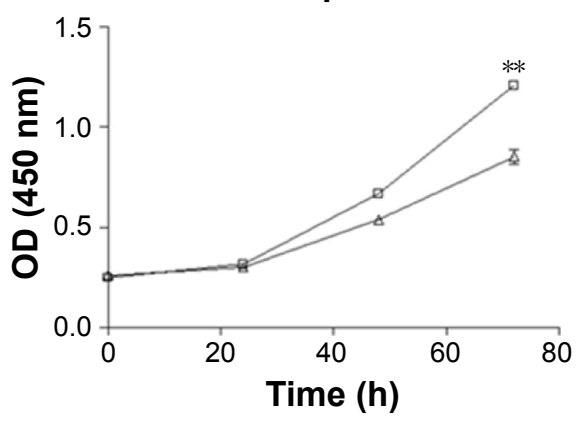

SMMC-7721

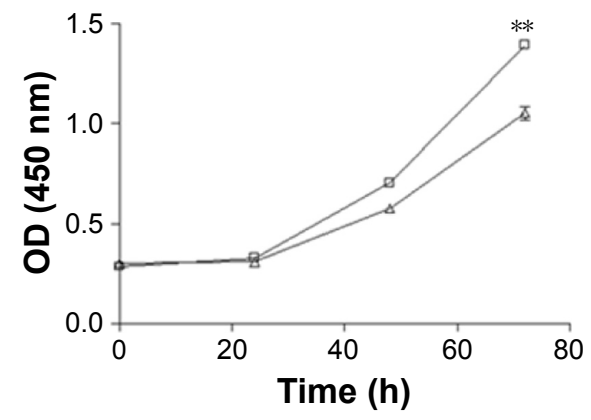

$\because \mathrm{si}-\mathrm{NC} \rightarrow \mathrm{si}-\mathrm{NEAT1}$

Figure 2 Knocking down NEATI inhibited the proliferation of the hepatocellular carcinoma cell lines HepG2 and SMMC-772I.

Notes: (A) HepG2 and SMMC-772I cells were treated with $100 \mathrm{nM}$ si-NEATI or si-NC for $6 \mathrm{~h}$. Total RNA was extracted $48 \mathrm{~h}$ later, and the expression levels of NEATI in each group were quantitatively analyzed and normalized to the GAPDH expression according to the $2^{-\Delta \Lambda \mathrm{ct}}$ method. The significance of differences between groups was measured with a paired-sample $t$-test. $* * * P<0.00$ I. (B) A total of $2 \times 10^{5} \mathrm{HepG} 2$ or SMMC-772I cells labeled with $2 \mu \mathrm{M}$ CFSE were seeded in six-well plates $48 \mathrm{~h}$ after siNEATI or si-NC treatment. The cells were collected, and the CFSE intensity was detected by flow cytometry to measure cell proliferation 0 and $72 \mathrm{~h}$ after seeding. The data represent at least three independent experiments. (C) A total of $2 \times 10^{3} \mathrm{HepG} 2$ and SMMC-772I cells were seeded in a $96-w e l l$ plate $48 \mathrm{~h}$ after si-NEATI or si-NC treatment. Ten microliters of CCK-8 was then added to each well $0,24,48$ and $72 \mathrm{~h}$ after seeding and the plate was incubated for $1 \mathrm{~h}$ at $37^{\circ} \mathrm{C}$. The cell viability was then assessed based on the OD450 value using an automatic microplate reader. Data are presented as the mean \pm SD from three independent experiments. The significance of differences between groups was measured with a two-way ANOVA. $* * P<0.01$.

Abbreviations: ANOVA, analysis of variance; CCK-8, cell counting kit-8; CFSE, cell tracer: carboxyfluorescein diacetate, succinimidyl ester; GAPDH, lyceraldehyde 3-phosphate dehydrogenase; hnRNP A2, heterogeneous nuclear ribonucleoprotein A2; NC, normal control; NEATI, nuclear-enriched abundant transcript I; OD, optical density; SD, standard deviation; si, small intefering. 

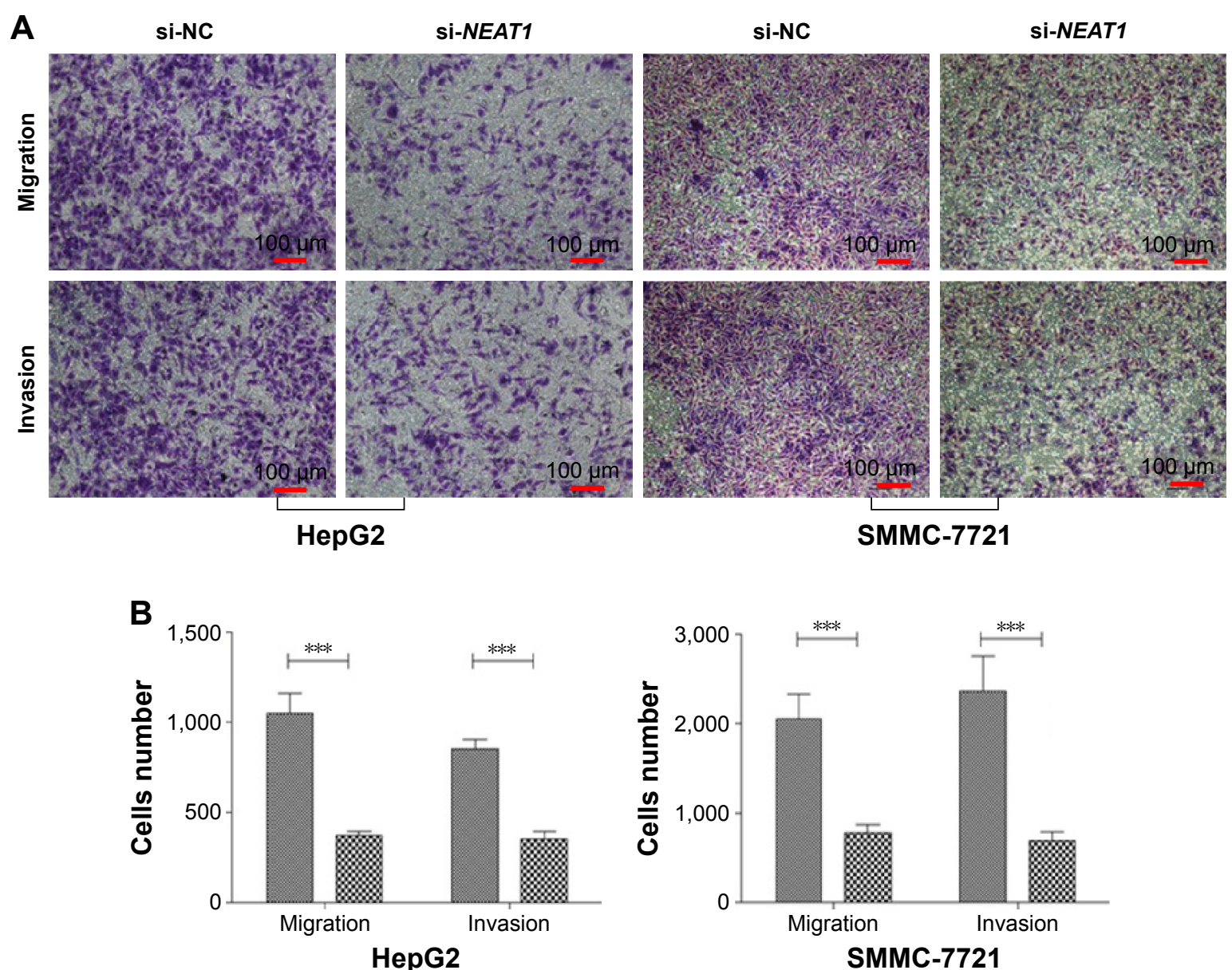

si-NC $\$ \mathbb{B}$ si-NEAT1

Figure 3 Knocking down NEATI inhibited the migration and invasion of the hepatocellular carcinoma cell lines HepG2 and SMMC-772I.

Notes: (A) A total of $2 \times 10^{5} \mathrm{HepG} 2$ and SMMC-772I cells treated with si-NEATI or si-NC were suspended in $200 \mu \mathrm{L}$ of serum-free DMEM in the upper chamber, which contained a porous membrane coated with Matrigel for the Transwell invasion assay; the membrane was uncoated for the migration assay. Subsequently, I5\% serum was added to the lower chamber as a chemoattractant. After migration for $24 \mathrm{~h}$ or invasion for $48 \mathrm{~h}$, cells that had penetrated the filters were fixed in dry methanol, stained with $0.1 \%$ crystal violet and then photographed. The scale bar represents $100 \mu \mathrm{m}$. (B) In each group, cells on the filter were detached with trypsin, and the number of cells was counted and is presented in the statistical graph. Data are presented as mean \pm SD from three independent experiments. Significant differences from each group were measured by paired sample $t$-test. $* * * p<0.001$.

Abbreviations: DMEM, Dulbecco's Modified Eagle's Medium; hnRNP A2, heterogeneous nuclear ribonucleoprotein A2; NC, normal control; NEATI, nuclear-enriched abundant transcript I; SD, standard deviation; si, small intefering.

cells compared with the si-NC group, as evidenced by crystal violet staining (Figure 3A). Moreover, significantly fewer cells detached from the Transwell filters in the si-NEAT1 group than in the si-NC group $(P<0.001$; Figure 3B).

\section{Knockdown of NEATI altered global gene expression patterns in HCC cells}

To study the molecular mechanism of NEAT1 knockdown, global transcriptional changes in HepG2 cells occurring $48 \mathrm{~h}$ after NEAT1-siRNA treatment were assessed by gene sequencing. Overall, 377 genes were differentially expressed in NEAT1 knockdown cells (including 229 upregulated and 148 downregulated genes, fold change $>2.0$ and $P$-value $<0.05)$. A gene ontology analysis showed that many differentially expressed genes are involved in the biologic processes and molecular functions relevant to cancer pathogenesis, such as cytokine-mediated signaling pathways, the activation of mitogen-activated protein kinase (MAPK) activity, the janus kinase (JAK)-signal transducers and activators of transcription (STAT) cascade (which is involved in the growth hormone signaling pathway), RNA splicing, the regulation of gene expression, RNA binding, cell proliferation and growth (Figure 4A). The associated biologic processes and molecular functions as shown in Figure 4A were the top ten variations after NEAT1 knockdown. The Kyoto Encyclopedia of Genes and Genomes pathway analysis identified variations in the tumor necrosis factor (TNF) signaling pathway, p53 signaling pathway, transcriptional 


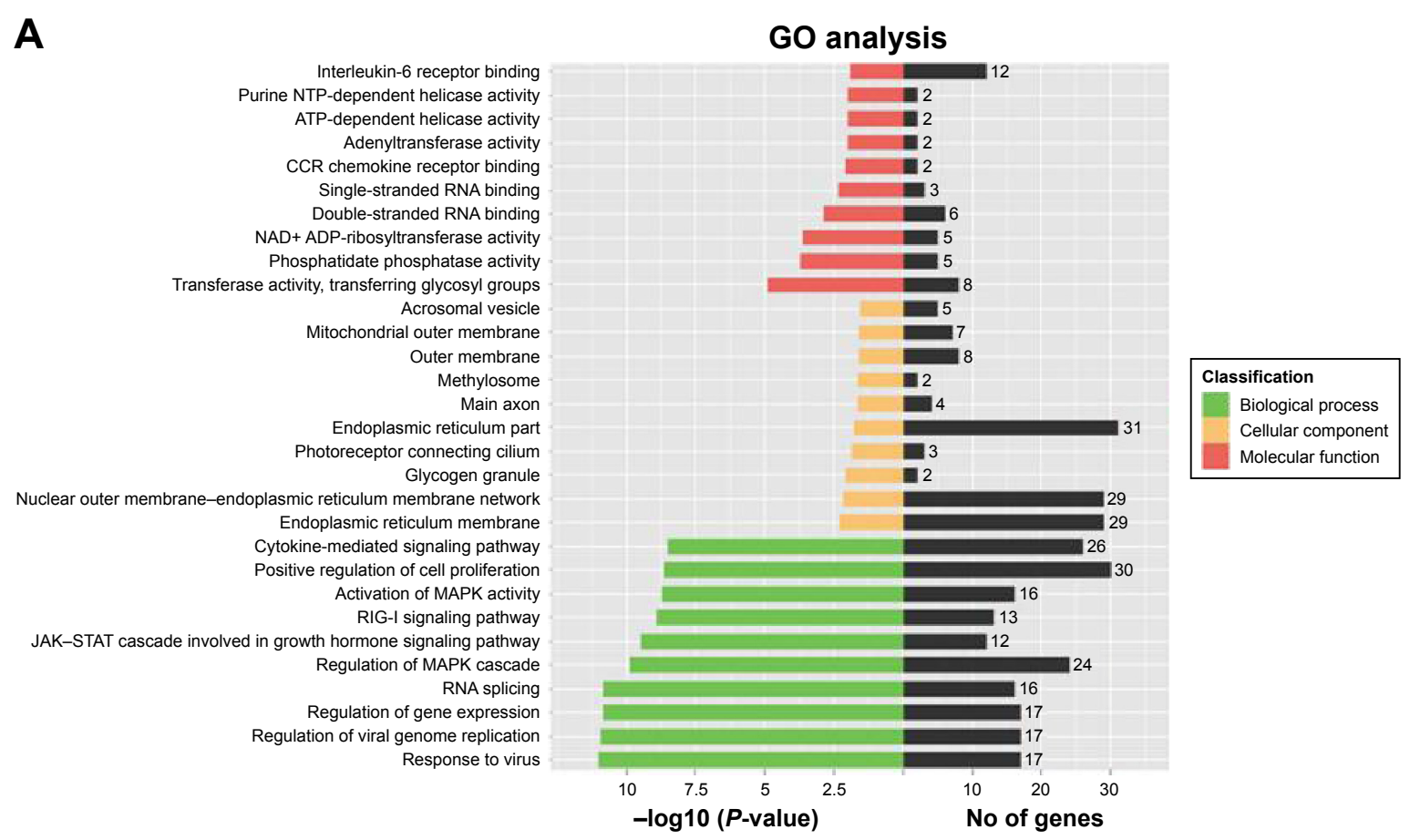

B
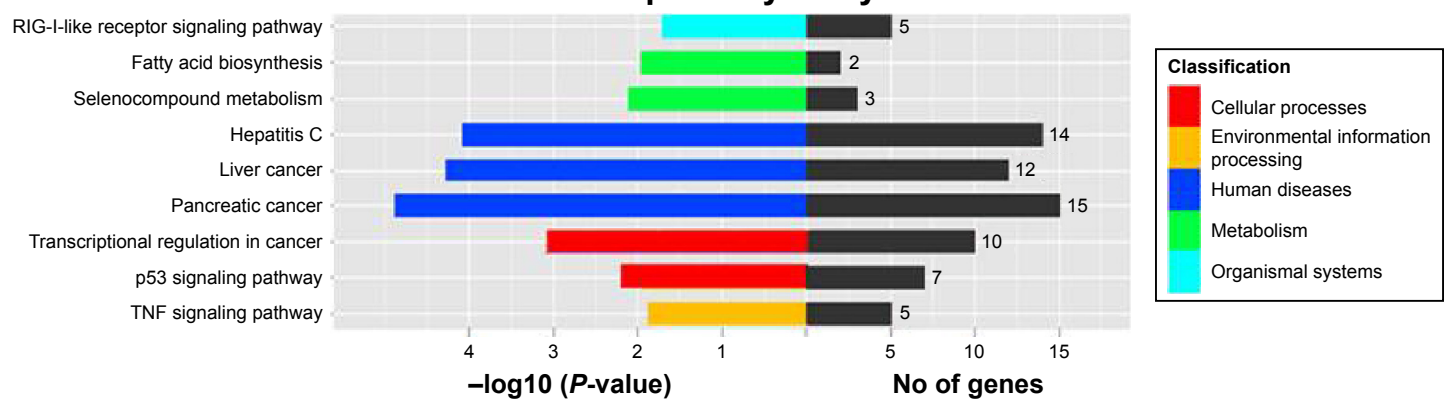

Figure 4 Knockdown of NEATI altered global gene expression patterns in HCC cells.

Notes: HepG2 cells were treated with si-NEATI for $6 \mathrm{~h}$, and RNA was extracted $48 \mathrm{~h}$ later. Gene sequencing assays were performed to screen for global transcriptional changes. (A) Hits for different genes and associated biologic processes and molecular function are presented based on the GO analysis of NEATI knockdown gene sequencing data. (B) Hits for different genes and associated pathways are presented based on the KEGG pathway analysis of NEATI knockdown gene sequencing data.

Abbreviations: CCR; C chemokine receptor; GO, gene ontology; HCC, hepatocellular carcinoma; hnRNP A2, heterogeneous nuclear ribonucleoprotein A2; KEGG, Kyoto Encyclopedia of Genes and Genomes; NC, normal control; NEATI, nuclear-enriched abundant transcript I; NTP, nucleoside triphosphate; si, small interfering.

regulation and RIG-I-like receptor signaling pathway after NEATl knockdown (Figure 4B). Based on the bioinformatics analysis and the fact that the overexpression of NEAT1 in HCC tissues and cell lines promoted HCC cell proliferation and invasion, we selected 13 genes, including six upregulated and seven downregulated genes, that are involved in the biologic processes relevant to cancer pathogenesis and have been reported as potential tumor suppressors or oncogenes in HCC (Table 1). ${ }^{8,25-35}$ Among these genes, we confirmed the downregulation of $h n R N P A 2$ and IQGAPI and the upregulation of STAT1, OSMR and IGFBP3 in HepG2 cells using RT-qPCR assays (Figure 5).

\section{The association of NEATI and U2AF65}

Several studies have found that many lncRNAs are involved in the molecular regulation pathways by interactions with RNA-binding proteins. ${ }^{5}$ Therefore, we hypothesized that NEAT1 might affect cellular functions in a similar manner. We screened starbase 2.0 protein-lncRNA interaction data and found that $\mathrm{U} 2 \mathrm{AF} 65$ protein has the highest clip read number associated with NEAT1. RIP assays were performed with U2AF65 antibody and nonspecific antibody (IgG control; Figure 6A) using extracts from HepG2 and SMMC-7721 cells to verify associated RNA enrichment. As shown in Figure 6B and C, NEATI enrichment (but not 
GAPDH mRNA enrichment) was observed in the U2AF65 antibody group, compared with the IgG control group. Primers against genes that were aberrantly expressed (Figure 5) were also used to detect enrichment using U2AF65 antibody. This experiment showed that mRNA $h n R N P A 2$ was significantly enriched using U2AF65 antibody (Figure 6B, C). However, a RIP assay using $h n R N P A 2$ antibody did not result in NEAT1 enrichment. We further performed an RNA pull-down assay using full-length NEAT1 ( $3^{\prime}$ end biotinylated) with extracts from HepG2 and SMMC-7721 cells to identify associated proteins. RNA-associated proteins were resolved by $10 \%$ sodium dodecyl sulfate polyacrylamide gel electrophoresis (Figure 6D), and bands associated with lncRNA-LET at the U2AF65, $\beta$-actin and hnRNP A2 locations (highlights 1, 2 and 3 in Figure 6D) were subjected to Western blot assays. Western blot assays using U2AF65, $\beta$-actin and $h n R N P A 2$ antibody identified a full-length NEAT1 band associated with U2AF65 protein, but not $\beta$-actin or $h n R N P A 2$ protein (Figure 6E). Several studies showed that some lncRNAs bind to RNA-binding proteins by a secondary structural element or a specific fragment. ${ }^{36}$ NEAT1 and U2AF65 interreaction data in starbase 2.0 showed target sites clip read numbers that were mostly located at the $3^{\prime}$ end and 5' end of 3,750 bp NEAT1. Therefore, five RNA fragments $(500-1,000 \mathrm{bp})$ of NEAT1 transcribed in vitro were 3' end biotinylated and used in RNA pull-down and Western blot assays with U2AF65 antibody. As shown in Figure 6F, U2AF65 protein was associated with fragments

Table I Selected transcriptional changes

\begin{tabular}{|c|c|c|}
\hline Selected gene name & Fold changes & $P$-value \\
\hline FASN (fatty acid synthase) & 0.352775678 & 0.000588873 \\
\hline TXNRDI (thioredoxin reductase I) & 0.421050498 & 0.009009959 \\
\hline$A R$ (androgen receptor) & 0.443871707 & 0.005314096 \\
\hline SRXNI (sulfiredoxin I) & 0.451058201 & 0.007842611 \\
\hline $\begin{array}{l}\text { hnRNP A2 (heterogeneous } \\
\text { nuclear ribonucleoprotein } A 2 / B I \text { ) }\end{array}$ & 0.478395062 & 0.016578563 \\
\hline FUS (FUS RNA-binding protein) & 0.483988604 & 0.011259985 \\
\hline IQGAPI (IQ motif containing & 0.498584211 & 0.014995828 \\
\hline GTPase activating protein I) & & \\
\hline DDX58 (DEXD/H-box helicase 58) & 4.630358625 & 0.000000012 \\
\hline $\begin{array}{l}\text { HERC5 (HECT and RLD domain } \\
\text { containing E3 ubiquitin protein ligase 5) }\end{array}$ & 3.336812332 & 0.000000271 \\
\hline $\begin{array}{l}\text { STATI (signal transducer and activator } \\
\text { of transcription I) }\end{array}$ & 3.668795232 & 0.004716635 \\
\hline OSMR (oncostatin M receptor) & 2.154985755 & 0.007187685 \\
\hline $\begin{array}{l}\text { NAMPT (nicotinamide } \\
\text { phosphoribosyltransferase) }\end{array}$ & 2.079423868 & 0.011377416 \\
\hline $\begin{array}{l}\text { IGFBP3 (insulin-like growth factor } \\
\text { binding protein 3) }\end{array}$ & 2.007540475 & 0.014632835 \\
\hline
\end{tabular}

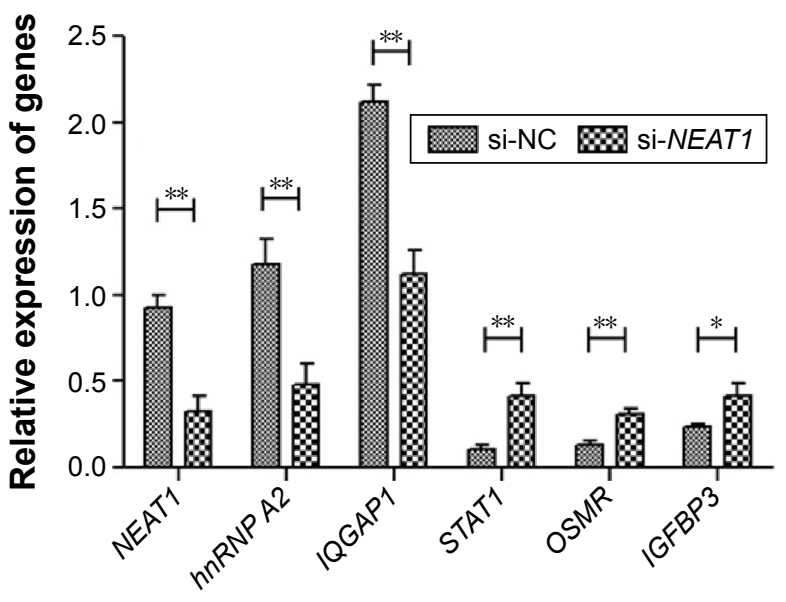

Figure 5 Knockdown of NEATI-regulated genes which were related with HCC progression.

Notes: The expression levels of genes selected for further validation were normalized to the expression of GAPDH according to the $2^{-\Delta \Delta C_{t}}$ method. The significance of differences between groups was assessed with a paired-sample $t$-test. $* P<0.05, * * P<0.01$. The data shown represent three independent experiments.

Abbreviations: GAPDH, lyceraldehyde 3-phosphate dehydrogenase; HCC, hepatocellular carcinoma; hnRNP A2, heterogeneous nuclear ribonucleoprotein A2; NEATI, nuclear-enriched abundant transcript I; si, small interfering.

2 and 5 of NEAT1, as detected by Western blot assays. The biotinylated RNA fragment 2 of NEAT1 was used in a subsequent EMSA because the signal intensity of this protein was higher than that of fragment 5 (Figure 6F).

\section{NEATI regulated $h n R N P A 2$ expression through U2AF65}

$\mathrm{U} 2 \mathrm{AF} 65$ is an essential splicing factor of polypyrimidine tract pre-mRNA ${ }^{37}$ and also known as a competitor of the hnRNP protein family in the regulation of transcript function. ${ }^{38}$ However, the role of U2AF65 in NEAT1-hnRNP $A 2$ regulation remains unknown. A Western blot assay and immunohistochemistry assays indicated that knocking down lncRNA NEAT1 downregulated $h n R N P A 2$ protein, but not U2AF65 protein expression in HepG2 cells (Figure 7A). According to RIP and RNA pull-down assays, NEAT1 did not directly bind to $h n R N P A 2$ protein. Moreover, EMSA showed that hnRNP mRNA competed with NEATI fragment 2 to bind proteins in HepG2 cell extracts (Figure 7B). We hypothesized that $N E A T 1$ regulates $h n R N P A 2$ expression through the NEAT1-U2AF65 complex. Therefore, U2AF65 was knocked down and $h n R N P A 2$ expression was detected by Western blot and immunohistochemistry assays. Western blot and immunohistochemistry assays indicated that knocking down U2AF65 also downregulated $h n R N P A 2$. According to these data, U2AF65 may participate in NEAT1-hnRNP A2 regulation (Figure 7C, D). 
A

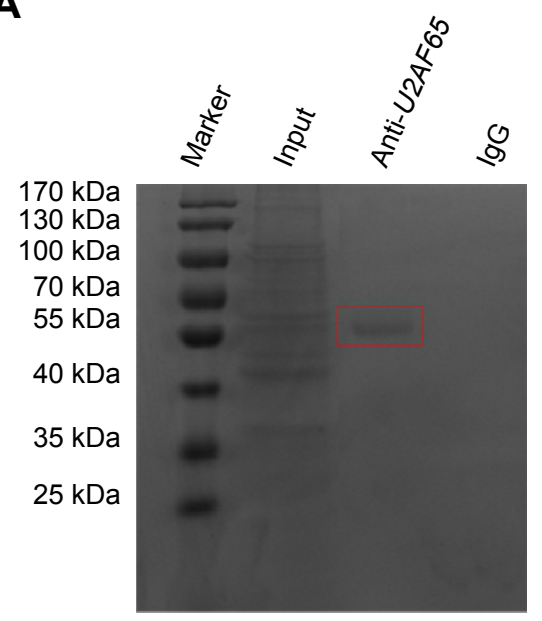

B

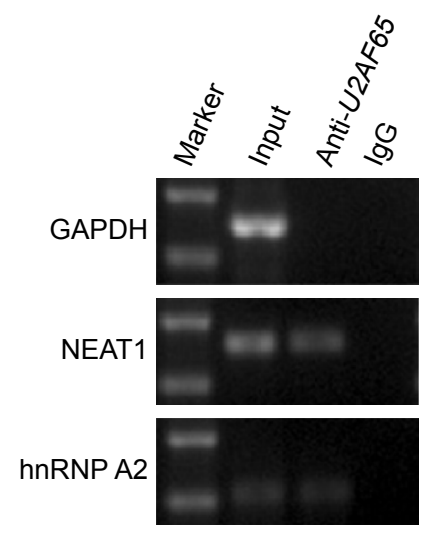

E

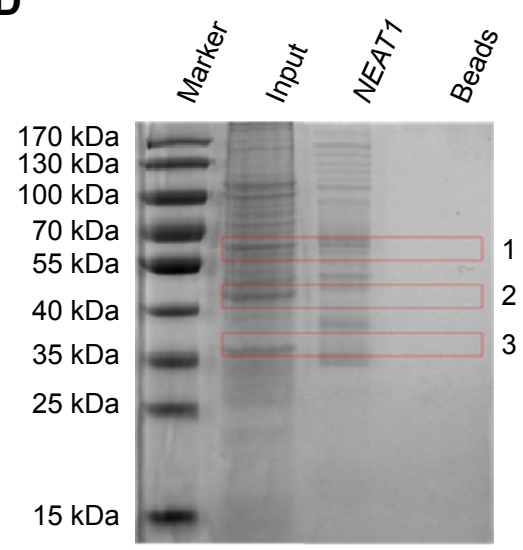

C

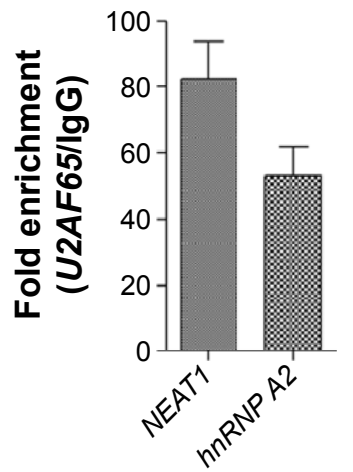

$\mathbf{F}$

Chr11:65,190,269-65.194,003
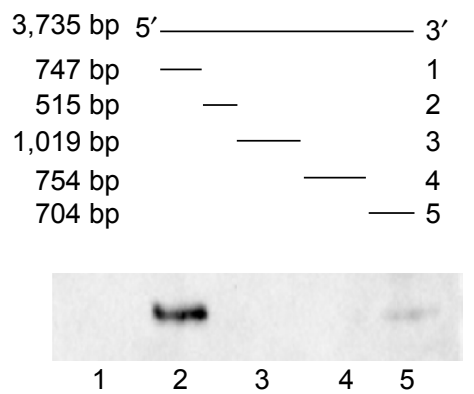

Figure 6 U2AF65 binds to NEATI and mRNA hnRNP A2.

Notes: (A) RIP experiments were performed using the U2AF65 antibody and IgG control antibody. HepG2 or SMMC-772I cell lysate (input control) was incubated with beads coated with U2AF65 antibody or IgG control antibody for $6 \mathrm{~h}$ at $4^{\circ} \mathrm{C}$. Proteins eluted from the beads with SDS in each group were subjected to gel electrophoresis. The gel was stained with Coomassie blue and photographed. The highlighted region represents U2AF65 protein. (B) Beads attached to an antibody-protein-RNA complex were incubated with proteinase $\mathrm{K}$ at $55^{\circ} \mathrm{C}$ for $30 \mathrm{~min}$, and the RNA was extracted by the TRlzol:chloroform method. RT-qPCR was then performed using specific primers to detect IncRNA-NEATI, hnRNPA2 or GAPDH. The RT-qPCR product of each group was subjected to electrophoresis and photographed. (C) The quantitative expression levels of NEATI and hnRNP A2 were normalized to GAPDH expression in each group according to the $2^{-\Delta \Delta C t}$ method. RIP enrichment was assessed based on RNA associated with the U2AF65 protein, which was compared with the lgG control group. The fold enrichment of NEATI and hnRNP A2 in the anti-U2AF65 group is presented in the statistical graph. The data represent the average and standard deviation of three independent experiments. (D) Full-length NEATI was biotinylated and targeted with streptavidin beads and then incubated with whole HepG2 extracts (input control) for I h at $4^{\circ} \mathrm{C}$ and washed. Streptavidin beads without biotinylated RNA are shown as a control. Associated protein eluted from the beads was subjected to gel electrophoresis, stained with Coomassie blue and photographed. (E) The highlighted regions were subjected to Western blotting using U2AF65 antibody for region I, $\beta$-actin antibody for region 2 and hnRNP A2 antibody for region 3 . The bands in each group were visualized by exposure for 20 $s$ under a CCD camera. (F) RNAs corresponding to the different fragments (I-5) of NEATI were biotinylated and incubated with HepG2 whole cell extracts, targeted with streptavidin beads and washed. Bound U2AF65 protein was detected by Western blotting $(n=3)$.

Abbreviations: CCD, charge coupled device; GAPDH, lyceraldehyde 3-phosphate dehydrogenase; hnRNP A2, heterogeneous nuclear ribonucleoprotein A2; IgG, immuglobulin G; NEATI, nuclear-enriched abundant transcript I; RIP, RNA immunoprecipitation; RT-qPCR, reverse transcription quantitative polymerase chain reaction; SDS, sodium dodecyl sulfate; U2AF65, U2 small nuclear RNA auxiliary factor 2.

\section{Overexpression of $h n R N P A 2$ rescued the proliferation and invasion in HCC cells that express low levels of NEATI}

$h n R N P A 2$ is an essential splicing factor that promotes HCC proliferation and invasion ${ }^{39}$ and activates alternative splicing switch that downregulates a dominant-negative isoform of A-Raf, which leads to activation of the Raf-MEK-ERK pathway and cellular transformation. ${ }^{22}$ Our data indicated that $N E A T 1$ participates in $h n R N P A 2$ regulation. To verify that $N E A T 1$ functions by $h n R N P A 2$ regulation, $h n R N P A 2$ was overexpressed in $N E A T 1$ knockdown cells using a retrovirus that encodes $h n R N P A 2$ (Figure 8A), as described previously. ${ }^{22}$ Control cells were transfected with an empty vector (lv-control). Subsequent proliferation and invasion assays indicated that the overexpression of $h n R N P A 2$ promoted the proliferation and invasion of NEATl knockdown 
A

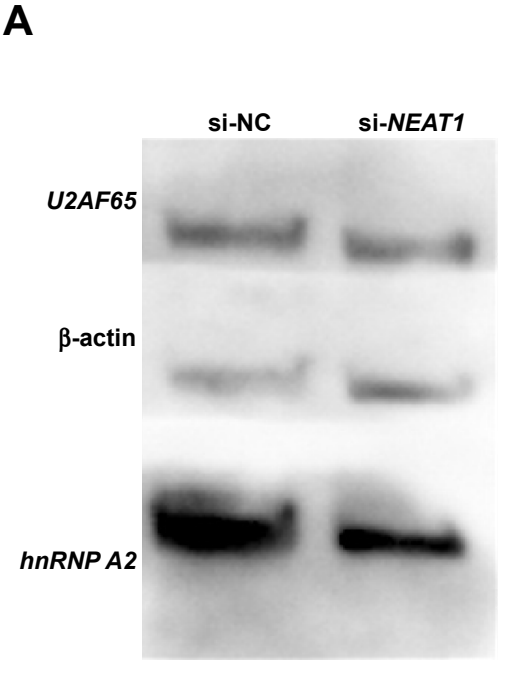

B

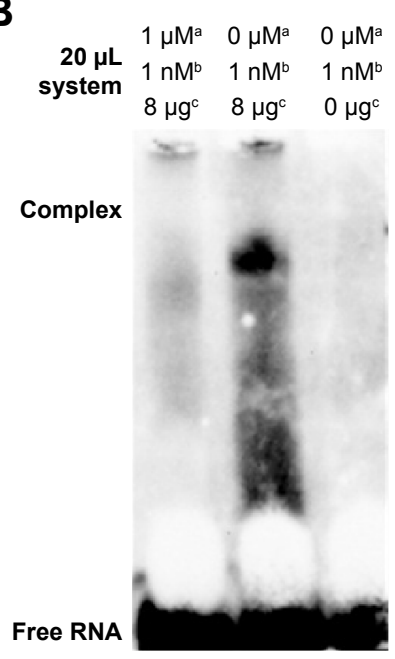

C

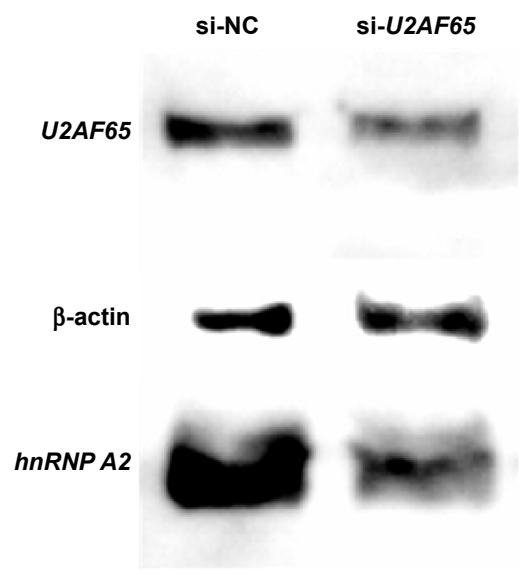

D

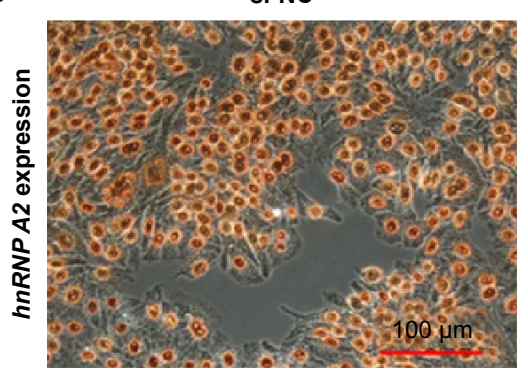

si-NC
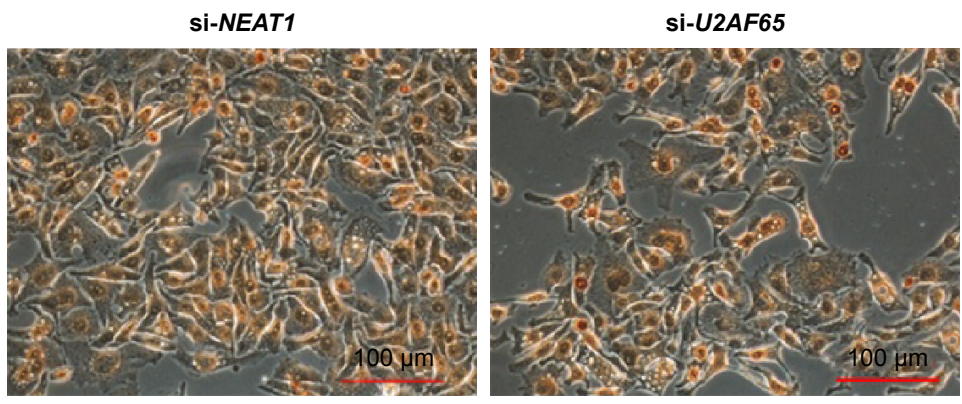

Figure 7 Knocking down NEATI and U2AF65 downregulated hnRNP A2 expression.

Notes: (A) NEATI was knocked down in HepG2 cells using si-NEATI or si-NC (control). HepG2 cell extracts were obtained $48 \mathrm{~h}$ after transfection. The extracts from each group were subjected to Western blotting to detect U2AF65, hnRNP A2 and $\beta$-actin. (B) An EMSA competition experiment was performed using I nM of the biotinylated IncRNA-NEATI fragment 2, a I,000-fold molar excess of unlabeled hnRNP A2 mRNA and whole HepG2 extracts in a total volume of $20 \mu \mathrm{L}$. Different ratios of biotinylated RNA, protein and unlabeled RNA were incubated. The masses of the $20 \mu \mathrm{L}$ system were resolved using $6 \%$ native polyacrylamide gel electrophoresis, and the proteins were

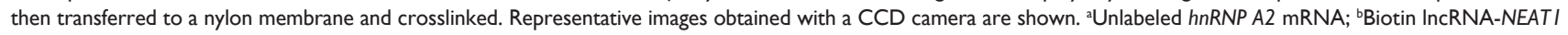
fragment 2; 'HepG2 extract. (C) U2AF65 mRNA was knocked down in HepG2 cells using si-U2AF65 or si-NC (control). HepG2 cell extracts were obtained 48 h after transfection. The extracts from each group were subjected to Western blotting to detect U2AF65, hnRNP A2 and $\beta$-actin. (D) Slides of HepG2 cells were incubated with hnRNP A2 antibody at $4^{\circ} \mathrm{C}$ for $12 \mathrm{~h}$ after si-NEATI, si-U2AF65 or si-NC treatment, and the slides were then incubated with secondary HRP-antibody, colored with a DAB kit and photographed. The scale bar represents $100 \mu \mathrm{m}$.

Abbreviations: CCD, charge coupled device; DAB, diaminobenzidine; EMSA, electrophoretic mobility shift assay; hnRNP A2, heterogeneous nuclear ribonucleoprotein A2; HRP, horseradish peroxidase; mRNA, messenger RNA; NC, normal control; NEATI, nuclear-enriched abundant transcript I; si, small interfering; U2AF65, U2 small nuclear RNA auxiliary factor 2 .

cells, compared with the lv-control group (Figure 8B-D). These data suggest that NEAT1 promoted HCC cell proliferation and invasion by regulating $h n R N P A 2$.

\section{Knockdown of NEATI reduced HepG2 cell growth and downregulated hnRNPA2 expression in vivo}

HepG2 cells were transfected with si-NEAT1 and expressed lower level of NEAT1 than the control cell line. Then the hnRNP A2 expression level was tested to explore NEAT1$h n R N P A 2$ regulation in vivo. Cells of either line were bilaterally injected into the axillary fossa of male athymic nude mice, and the tumor growth activity and $h n R N P A 2$ expression were measured. As shown in Figure 9A, the average volume of tumors derived from the si-NEAT1 group 14 days after xenograft transplantation was significantly smaller than that of the control group ( $\mathrm{n}=4$ animals per group, $P<0.001)$. Furthermore, total RNA was also extracted from the tumors in each group to measure the expression level of $h n R N P A 2$ by RT-qPCR. The results revealed that the expression of $h n R N P A 2 \mathrm{mRNA}$ was significantly lower in the si-NEAT1 group than in the control group ( $P<0.001$; Figure 9B). Furthermore, Western blot assays indicated that the protein expression of $h n R N P A 2$ was decreased in the si-NEAT1 group compared with the control group (Figure 9C); a representative immunohistochemistry image is shown in Figure 9D. 
A

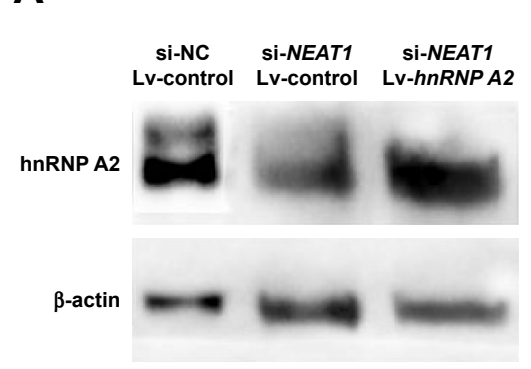

D

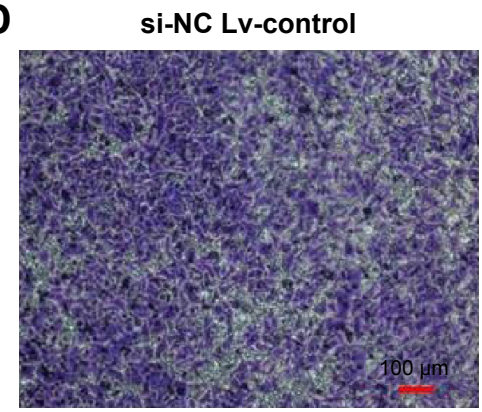

B

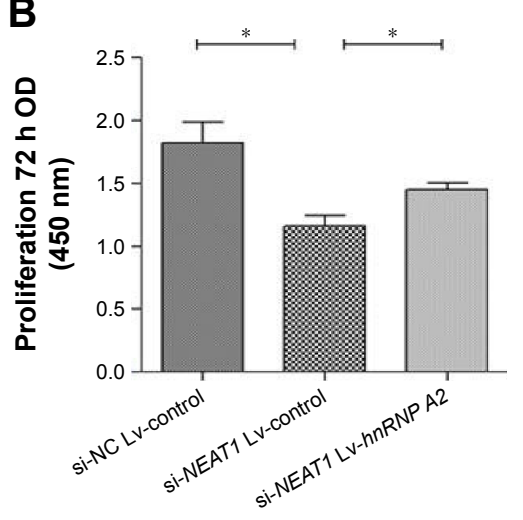

si-NEAT1 Lv-control

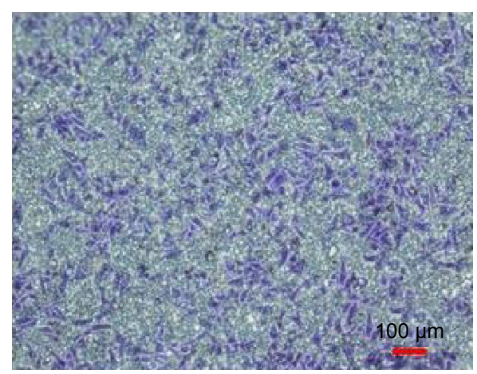

C

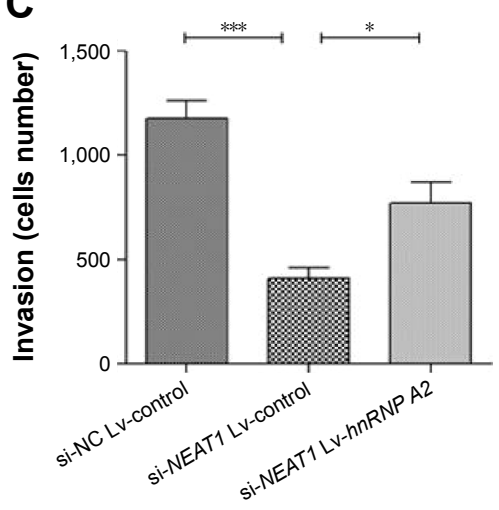

si-NEAT1 Lv-hnRNP A2

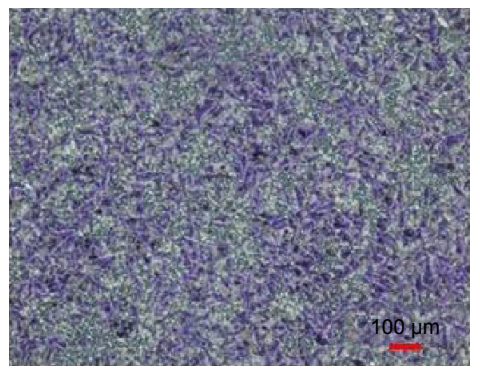

Figure 8 The overexpression of hnRNP A2 rescued the proliferation and invasion of HCC cells expressing low levels of IncRNA-NEATI.

Notes: (A) NEATI was knocked down in HepG2 cells using si-NEATI or si-NC (control). The overexpression of hnRNP A2 in cells transfected with retroviruses encoding empty vector or hnRNPA2 was analyzed by Western blotting for hnRNP A2. $\beta$-actin was used as a loading control. (B) The cells from each group described in A were seeded in 96-well plates and cultured with $100 \mu \mathrm{L}$ of DMEM containing $10 \%$ serum for $72 \mathrm{~h}$. Ten microliters of CCK-8 was added to each well and incubated for I h at $37^{\circ} \mathrm{C}$. The cell viability was then assessed based on the OD450 value using an automatic microplate reader. Number (C) and representative images (D) of invasive cells per filter in each group described in A. Transwell assays were performed according to the method described in Figure 2D. The significance of differences between groups was assessed with a one-way ANOVA followed by Dunnett's multiple comparison post hoc test. $* P<0.05$, $* * * P<0.01$.

Abbreviations: ANOVA, analysis of variance; CCK-8, cell counting kit-8; DMEM, Dulbecco's Modified Eagle's Medium; HCC, hepatocellular carcinoma; hnRNPA2, heterogeneous nuclear ribonucleoprotein A2; IncRNA, long noncoding RNAs; NC, normal control; NEATI, nuclear-enriched abundant transcript I; OD, optical density; si, small interfering.

\section{Discussion}

According to a previous study, NEAT1 expression is higher in cancers than in normal tissues, including leukemia, glioma, non-small cell lung cancer, prostate cancer, breast cancer, ovarian carcinoma and HCC. NEATl expression was associated with metastasis-associated lung adenocarcinoma transcript 1 (MALAT1), which is a well-known oncogene because it regulates the alternative splicing of endogenous target genes involved in cancers, including HCC. ${ }^{21}$ The biologic mechanisms of NEAT1 regulation in cancer have gradually been revealed in recent studies. In prostate cancer, NEAT1 is involved in the modulation of oncogenic growth by altering the epigenetic landscape. ${ }^{18}$ Induced by hypoxia in hypoxia-inducible factors transcriptional pathways, NEATl also plays an important role in promoting proliferation and reducing apoptosis, which contributes to tumorigenesis. ${ }^{40} \mathrm{In}$ glioma, NEAT1 regulates glioma cell proliferation, invasion and migration through the miR-449b-5p/c-Met axis. ${ }^{14}$ Moreover, a study of HCC patients indicated that the expression level of $N E A T 1$ strongly correlated with tumorigenesis and metastasis, including the number of tumor nodes, metastasis, portal vein tumor embolus, vascular invasion, tumor capsular infiltration and TNM stage. ${ }^{22}$ However, the specific functions, regulatory roles and biologic mechanisms of NEAT1 in HCC remained unclear. We verified that NEAT1 is upregulated in HCC tissues and cell lines and affects HCC (Figure 1). Further study indicated that NEATI promotes cell proliferation, migration and invasion in the HCC cell lines HepG2 and SMMC-7721 (Figures 2 and 3). RNA sequencing and data analyses indicated that NEATI plays important roles in the regulation of pathways and genes in HCC cells (Figure 4). We also verified that $h n R N P A 2$ and IQGAPI were downregulated and that STAT1, OSMR and IGFBP3 were upregulated in HCC cells (Figure 5).

LncRNA-protein complexes have been shown to initiate unique transcriptional programs that result in various functions by different combinations of RNA-binding protein interactions. ${ }^{5}$ Specific hypothesis-driven studies confirmed this theory in HCC. LncRNA-LET, a tumor suppressor, binds to NF90, to affect hypoxia-inducible factor-1a mRNA 

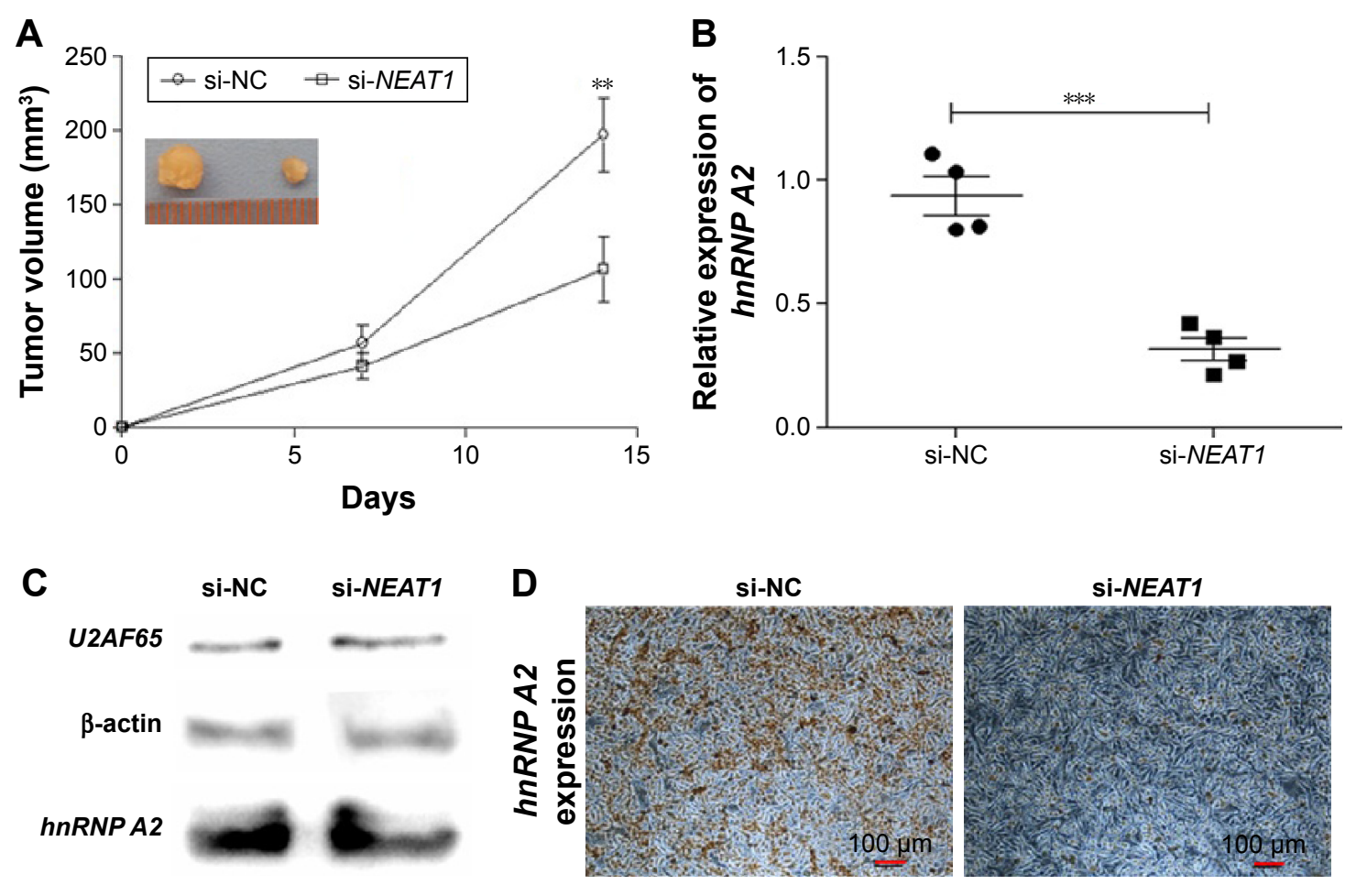

Figure 9 Knocking down NEATI inhibited HepG2 cell growth and downregulated hnRNP A2 expression in vivo.

Notes: (A) The axillary fossae of male athymic nude mice aged 4-6 weeks were bilaterally inoculated with $2 \times 10^{6}$ cells $/ 0.1 \mathrm{~mL}$ per site. HepG 2 cells were transfected with si-NEATI and expressed a lower level of NEATI than the control group. Tumors were taken and size was monitored 7 and I4 days later ( $\mathrm{n}=4$ ). $* * P<0.0 \mathrm{I}$. (B) Total RNA was extracted from the tumors 14 days later and the expression levels of hnRNP A2 in each group were quantitatively analyzed by normalizing them to the GAPDH level according to the $2^{-\Delta \Delta C t}$ method $(n=3)$. ${ }^{* * *} P<0.001$. (C) The tumor extracts from each group were subjected to a Western blot analysis to detect $U 2 A F 65$, hnRNP A2 and $\beta$-actin. (D) A representative IHC image obtained using hnRNP A2 antibody as the primary antibody is shown. The scale bar represents $100 \mu \mathrm{m}$.

Abbreviations: GAPDH, lyceraldehyde 3-phosphate dehydrogenase; hnRNP A2, heterogeneous nuclear ribonucleoprotein A2; IHC, immunohistochemistry; NC, normal control; NEATI, nuclear-enriched abundant transcript I; si, small interfering; U2AF65, U2 small nuclear RNA auxiliary factor 2.

accumulation and stability under hypoxic conditions in HCC. ${ }^{7}$ LncRNA-MEG3 $3{ }^{41}$ IncRNA Rs $10680577,{ }^{42}$ LncRNA-HEIH $^{43}$ and HOTTIP ${ }^{44}$ function through lncRNA-protein complexes according to reports. Because NEAT1 plays important roles in promoting the proliferation and invasion of HCC cells and is involved in gene regulation, we screened the RNAprotein interaction data in starbase 2.0. Specifically, we searched for proteins that were either up- or downregulated in NEAT1 knockdown cells (Figure 3C) and determined whether they directly bound to NEATI. However, the starbase 2.0 data identified few possible interactions between NEAT1 and the proteins we selected based on the clip read number. Therefore, we hypothesized that $N E A T l$ functions by gene regulation, which might be associated with the NEAT1-RNA-binding protein complex. We screened the RNA-protein interaction data in starbase 2.0 and found that $\mathrm{U} 2 \mathrm{AF} 65$, polypyrimidine tract-binding protein (PTB), eukaryotic initiation factor 4A-III (eIF4AIII) and fused in sarcoma (FUS) might interact with NEAT1. Using RIP and RNA pull-down assays, we found that NEAT1 binds to U2AF65 protein and that the binding target might be located in NEAT1 fragments 2 (chr11: 65, 191, 001-65, 191, 516) and 5 (chr11: 65, 193, 299-65, 194, 003) in vitro (Figure 6). Furthermore, a RIP assay showed that hnRNP A2 mRNA was enriched by U2AF65 protein (Figure 6). This finding suggested that U2AF65 interacts with $h n R N P A 2$ mRNA, which was downregulated in $N E A T 1$-knockdown HCC cells. $h n R N P A 2$ was reported to be an essential splicing factor that promotes cell proliferation and invasion and correlates with poor outcome in HCC patients. ${ }^{22,39}$ Specifically, this factor activates the Raf-MEK-ERK pathway, which results in cellular transformation in HCC..$^{40}$ A previous study indicated that lncRNA functions through a specific region which was the combination target of RNA-protein. ${ }^{8}$ Because U2AF65 protein interacts with $N E A T 1$ and $h n R N P A 2$ mRNA, the NEAT1-U2AF65-hnRNP A2 complex was further explored in HCC cells. Our RNA pull-down data suggested that NEAT1 directly binds to U2AF65 protein, but not $h n R N P A 2$ protein (Figure 6). Knocking down NEAT1 or U2AF65 reduced the downregulation of hnRNP A2 transcript and protein. An EMSA showed NEAT1 fragment 2 (chr11: 65, 191, 001-65, 191, 516), which specifically interacts with 
U2AF65, competes with $h n R N P A 2$ mRNA to bind proteins in vitro. Our future studies will focus on the specific region that regulates the function of NEAT1 in HCC. Our updated data obtained using recombinant protein will confirm that NEAT1 competes with $h n R N P A 2$ to bind U2AF65 protein. Furthermore, overexpression assays will be used to confirm that NEAT1 fragment 2 (chr11: 65, 191, 001-65, 191, 516) and the $515 \mathrm{nt}$ region regulate $h n R N P A 2$ and tumorigenesis in HCC. Taken together, our data indicate that NEAT1 regulates $h n R N P A 2$ expression in $\mathrm{HCC}$ cells, and this regulation might be associated with the NEAT1-U2AF65 protein complex.

NEAT1 has been previously described to function by regulating the epigenetic landscape. ${ }^{14,18,21}$ As demonstrated above, knocking down NEAT1 decreases $h n R N P A 2$ expression, which is strongly correlated with HCC tumorigenesis. However, whether NEAT1 promotes HCC cell proliferation and invasion by regulating $h n R N P A 2$ regulation remains to be verified. To explore the correlation between NEAT1 and $h n R N P A 2$ in HCC tumorigenesis, we compared the proliferative and invasive potential of si-NEAT1-transfected and si-NEAT1 and $h n R N P A 2$-cotransfected cells. Our data indicated that the overexpression of $h n R N P A 2$ rescued the proliferation and invasion inhibited by NEAT1 knockdown in HCC cells (Figure 8). Because NEAT1 was also found to regulate hnRNP A2 expression, this finding suggested that NEAT1 might promote cell proliferation and invasion by regulating $h n R N P A 2$.

Analyses of clinical pathologic data, in vitro assays based on knockdown/knockout or overexpression models in cell lines and in vivo xenograft models have confirmed that lncRNAs regulate tumorigenesis and metastasis. ${ }^{36}$ Therefore, we compared the tumor growth of NEAT1-knockdown cells and control cells in athymic nude mice. Our data indicated that knocking down NEAT1 inhibited tumor growth in vivo. Interestingly, $h n R N P A 2$ expression was downregulated in the tumors of the NEAT1 knockdown group. These data suggested that NEAT1 promotes tumor growth and affects hnRNP $A 2$ in vivo.

In summary, this work suggests that the lncRNA NEAT1 is an oncogene and is strongly correlated with altered $h n R N P$ $A 2$ expression. Thus, NEAT1-hnRNP A2 regulation might be a potential mechanism of HCC progression.

\section{Acknowledgments}

The authors would like to thank Dr Lei Zhang from the Biomedical Research Center of The Calmette Affiliated Hospital of Kunming Medical University, The First Hospital of Kunming, for helping us with molecular experiments.

\section{Disclosure}

This study was supported by grants to Li Li from Kunming Science and Technology Bureau (2012-02-03-A-H-04-0001). The other authors report no conflicts of interest in this work.

\section{References}

1. Ding J, Lu Q, Quyang Y, et al. A long noncoding RNA regulates photoperiod-sensitive male sterility, an essential component of hybrid rice. Proc Natl Acad Sci U S A. 2012;109(7):2654-2659.

2. Calvisi DF, Ladu S, Pinna F, et al. SKP2 and CKS1 promote degradation of cell cycle regulators and are associated with hepatocellular carcinoma prognosis. Gastroenterology. 2009;137(5):1816-1826, e1-e10.

3. Jendrzejewski J, He H, Radomska HS, et al. The polymorphism rs 944289 predisposes to papillary thyroid carcinoma through a large intergenic noncoding RNA gene of tumor suppressor type. Proc Natl Acad Sci US A. 2012;109(22):8646-8651.

4. Spizzo R, Almeida MI, Colombatti A, Calin GA. Long non-coding RNAs and cancer: a new frontier of translational research? Oncogene. 2012;31(43):4577-4587.

5. Guttman M, Donaghey J, Carey BW, et al. lincRNAs act in the circuitry controlling pluripotency and differentiation. Nature. 2011;477(7364): 295-300.

6. Kogo R, Shimamura T, Mimori K, et al. Long noncoding RNA HOTAIR regulates polycomb-dependent chromatin modification and is associated with poor prognosis in colorectal cancers. Cancer Res. 2011;71(20): 6320-6326.

7. Yang F, Huo XS, Yuan SX, et al. Repression of the long noncoding RNA-LET by histone deacetylase 3 contributes to hypoxia-mediated metastasis. Mol Cell. 2013;49(6):1083-1096.

8. Mohamadkhani A. Long noncoding RNAs in interaction with RNA binding proteins in hepatocellular carcinoma. Hepat Mon. 2014;14(5): e18794.

9. Huang JL, Zheng L, Hu YW, Wang Q. Characteristics of long noncoding RNA and its relation to hepatocellular carcinoma. Carcinogenesis. 2014;35(3):507-514.

10. Naganuma T, Hirose T. Paraspeckle formation during the biogenesis of long non-coding RNAs. RNA Biol. 2013;10(3):456-461.

11. Guru SC, Agarwal SK, Manickam P, et al. A transcript map for the 2.8-Mb region containing the multiple endocrine neoplasia type 1 locus. Genome Res. 1997;7(7):725-735.

12. Clemson CM, Hutchinson JN, Sara SA, et al. An architectural role for a nuclear noncoding RNA: NEAT1 RNA is essential for the structure of paraspeckles. Mol Cell. 2009;33(6):717-726.

13. Zeng $\mathrm{C}, \mathrm{Xu} \mathrm{Y}, \mathrm{Xu} \mathrm{L}$, et al. Inhibition of long non-coding RNA NEAT1 impairs myeloid differentiation in acute promyelocytic leukemia cells. BMC Cancer. 2014;14:693.

14. Zhen L, Yun-Hui L, Hong-Yu D, Jun M, Yi-Long Y. Long noncoding RNA NEAT1 promotes glioma pathogenesis by regulating miR-449b5p/c-Met axis. Tumour Biol. 2016;37(1):673-683.

15. Pan LJ, Zhong TF, Tang RX, et al. Upregulation and clinicopathological significance of long non-coding NEAT1 RNA in NSCLC tissues. Asian Pac J Cancer Prev. 2015;16(7):2851-2855.

16. Ke H, Zhao L, Feng X, et al. NEAT1 is required for survival of breast cancer cells through FUS and miR-548. Gene Regul Syst Bio. 2016; 10(Suppl 1):11-17.

17. Kim YS, Hwan JD, Bae S, Bae DH, Shick WA. Identification of differentially expressed genes using an annealing control primer system in stage III serous ovarian carcinoma. BMC Cancer. 2010;10:576.

18. Chakravarty D, Sboner A, Nair SS, et al. The oestrogen receptor alpharegulated lncRNA NEAT1 is a critical modulator of prostate cancer. Nat Commun. 2014;5:5383.

19. Cooper DR, Carter G, Li P, Patel R, Watson JE, Patel NA. Long non-coding RNA NEAT1 associates with SRp40 to temporally regulate PPARgamma2 splicing during adipogenesis in 3T3-L1 cells. Genes (Basel). 2014;5(4):1050-1063. 
20. Hirose T, Virnicchi G, Tanigawa A, et al. NEAT1 long noncoding RNA regulates transcription via protein sequestration within subnuclear bodies. Mol Biol Cell. 2014;25(1):169-183.

21. Guo S, Chen W, Luo Y, et al. Clinical implication of long non-coding RNA NEAT1 expression in hepatocellular carcinoma patients. Int $J$ Clin Exp Pathol. 2015;8(5):5395-5402.

22. Shilo A, Ben Hur V, Denichenko P, et al. Splicing factor hnRNP A2 activates the Ras-MAPK-ERK pathway by controlling A-Raf splicing in hepatocellular carcinoma development. RNA. 2014;20(4):505-515.

23. Huang G, Hu Z, Li M, et al. ECRG2 inhibits cancer cell migration, invasion and metastasis through the down-regulation of uPA/plasmin activity. Carcinogenesis. 2007;28(11):2274-2281.

24. Wan HY, Guo LM, Liu T, Liu M, Li X, Tang H. Regulation of the transcription factor NF-kappaB1 by microRNA-9 in human gastric adenocarcinoma. Mol Cancer. 2010;9:16.

25. Hu J, Che L, Li L, et al. Co-activation of AKT and c-Met triggers rapid hepatocellular carcinoma development via the mTORC1/FASN pathway in mice. Sci Rep. 2016;6:20484.

26. Tian YE, Xie XU, Lin Y, Tan G, Zhong WU. Androgen receptor in hepatocarcinogenesis: Recent developments and perspectives. Oncol Lett. 2015;9(5):1983-1988.

27. Tanase AM, Marchio A, Dumitrascu T, et al. Mutation spectrum of hepatocellular carcinoma from eastern-European patients betrays the impact of a complex exposome. J Expo Sci Environ Epidemiol. 2015; 25(3):256-263.

28. Mizuno H, Honda M, Shirasaki T, et al. Heterogeneous nuclear ribonucleoprotein A2/B1 in association with hTERT is a potential biomarker for hepatocellular carcinoma. Liver Int. 2012;32(7):1146-1155.

29. Jin X, Liu Y, Liu J, et al. The overexpression of IQGAP1 and betacatenin is associated with tumor progression in hepatocellular carcinoma in vitro and in vivo. PLoS One. 2015;10(8):e0133770.

30. Liu Z, Dou C, Jia Y, et al. RIG-I suppresses the migration and invasion of hepatocellular carcinoma cells by regulating MMP9. Int J Oncol. 2015;46(4):1710-1720.

31. Xue F, Higgs BW, Huang J, et al. HERC5 is a prognostic biomarker for post-liver transplant recurrent human hepatocellular carcinoma. J Transl Med. 2015;13:379.

32. Chen J, Wang H, Wang J, Huang S, Zhang W. STAT1 inhibits human hepatocellular carcinoma cell growth through induction of p53 and Fbxw7. Cancer Cell Int. 2015;15:111.
33. Ehlting $\mathrm{C}$, Böhmer O, Hahnel MJ, et al. Oncostatin M regulates SOCS3 mRNA stability via the MEK-ERK1/2-pathway independent of p38(MAPK)/MK2. Cell Signal. 2015;27(3):555-567.

34. Lin YC, Wu HC, Liao CC, Chou YC, Pan SF, Chiu CM. Secretion of one adipokine Nampt/Visfatin suppresses the inflammatory stressinduced NF-kappaB activity and affects Nampt-dependent cell viability in Huh-7 cells. Mediators Inflamm. 2015;2015:392471.

35. Han JJ, Xue DW, Han QR, et al. Induction of apoptosis by IGFBP3 overexpression in hepatocellular carcinoma cells. Asian Pac J Cancer Prev. 2014;15(23):10085-10089.

36. Tsai MC, Spitale RC, Chang HY. Long intergenic noncoding RNAs: new links in cancer progression. Cancer Res. 2011;71(1):3-7.

37. Agrawal AA, McLaughlin KJ, Jenkins JL, Kielkopf CL. Structureguided U2AF65 variant improves recognition and splicing of a defective pre-mRNA. Proc Natl Acad Sci U S A. 2014;111(49):17420-17425.

38. Zarnack K, König J, Tajnik M, et al. Direct competition between hnRNP C and U2AF65 protects the transcriptome from the exonization of Alu elements. Cell. 2013;152(3):453-466.

39. Cui H, Wu F, Sun Y, Fan G, Wang Q. Up-regulation and subcellular localization of hnRNP A2/B1 in the development of hepatocellular carcinoma. BMC Cancer. 2010;10:356.

40. Karreth FA, Tay Y, Perna D, et al. In vivo identification of tumorsuppressive PTEN ceRNAs in an oncogenic BRAF-induced mouse model of melanoma. Cell. 2011;147(2):382-395.

41. Chang L, Wang G, Jia T, et al. Armored long non-coding RNA MEG3 targeting EGFR based on recombinant MS2 bacteriophage virus-like particles against hepatocellular carcinoma. Oncotarget. 2016;7(17): 23988-24004.

42. Zhu Z, Gao X, He Y, et al. An insertion/deletion polymorphism within RERT-IncRNA modulates hepatocellular carcinoma risk. Cancer Res. 2012;72(23):6163-6172.

43. Yang F, Zhang L, Huo XS, et al. Long noncoding RNA high expression in hepatocellular carcinoma facilitates tumor growth through enhancer of zeste homolog 2 in humans. Hepatology. 2011;54(5):1679-1689.

44. Quagliata L, Matter MS, Piscuoglio S, et al. Long noncoding RNA HOTTIP/HOXA13 expression is associated with disease progression and predicts outcome in hepatocellular carcinoma patients. Hepatology. 2014;59(3):911-923.
OncoTargets and Therapy

\section{Publish your work in this journal}

OncoTargets and Therapy is an international, peer-reviewed, open access journal focusing on the pathological basis of all cancers, potential targets for therapy and treatment protocols employed to improve the management of cancer patients. The journal also focuses on the impact of management programs and new therapeutic agents and protocols on

\section{Dovepress}

patient perspectives such as quality of life, adherence and satisfaction The manuscript management system is completely online and includes a very quick and fair peer-review system, which is all easy to use. Visit http://www.dovepress.com/testimonials.php to read real quotes from published authors. 